\title{
Lung Metastases in Newly Diagnosed Hypo-pharyngeal Cancer: A Population-Based Study
}

\author{
Na Shen ${ }^{1}$, Tao Li², Lei Zhou ${ }^{1}$, and Xu Zhou ${ }^{1}$ \\ ${ }^{1}$ Zhongshan Hospital Fudan University \\ ${ }^{2}$ Peking University Third Hospital
}

November 5, 2020

\begin{abstract}
Objectives: To characterize the incidence proportions and survival probability of patients with hypo-pharyngeal cancer and lung metastases. Design: Secondary data analysis Setting: The Surveillance, Epidemiology, and End Results (SEER) database of the National Cancer Institute. Participants: We identified 2814 adult patients diagnosed with hypo-pharyngeal cancer between 2010 and 2014 for whom the status of lung metastases was known. Patients with an unknown follow-up were excluded; leaving 2714 patients in this cohort. Main outcome measures: Multivariable logistic and Cox regression models were performed to identify the risk factors associated with the presence of lung metastases at diagnosis and five-year all-cause mortality respectively. Results: We identified 128 patients with lung metastases at the time of diagnosis of hypo-pharyngeal cancer, representing $4.7 \%$ of the entire cohort. Females were less likely to have lung metastases $(15,11.7 \%)(\mathrm{P}=0.037)$. Incidences of lung metastases was higher among patients with histological grade III/IV $(56,43.8 \%)(\mathrm{P}<0.001)$. Patients with lung metastases had a larger tumor size $(49.9690 .25 \mathrm{~mm}, \mathrm{P}<0.001)$. For each $10 \mathrm{~mm}$ increase in tumor size, the odds of having lung metastases increased by $6.6 \%$. Patients with lung metastases had a shorter survival time(HR:3.05, 95\% CI:2.17-4.28, P<0.001). For age, tumor size increase and black study subjects were also significantly associated with a shorter survival time. Conclusion: Our study provides insight into the epidemiology of lung metastases in patients with hypo-pharyngeal cancer. When the tumor is diagnosed, we should pay close attention to the sex, race, tumor size and histological grade in order to quickly detect the distant metastases.
\end{abstract}

\section{Lung Metastases in Newly Diagnosed Hypo-pharyngeal Cancer: A Population-Based Study}

\section{Abstract}

Objectives: To characterize the incidence proportions and survival probability of patients with hypopharyngeal cancer and lung metastases.

Design: Secondary data analysis

Setting: The Surveillance, Epidemiology, and End Results (SEER) database of the National Cancer Institute.

Participants: We identified 2814 adult patients diagnosed with hypo-pharyngeal cancer between 2010 and 2014 for whom the status of lung metastases was known. Patients with an unknown follow-up were excluded; leaving 2714 patients in this cohort.

Main outcome measures: Multivariable logistic and Cox regression models were performed to identify the risk factors associated with the presence of lung metastases at diagnosis and five-year all-cause mortality respectively.

Results: We identified 128 patients with lung metastases at the time of diagnosis of hypo-pharyngeal cancer, representing $4.7 \%$ of the entire cohort. Females were less likely to have lung metastases $(15,11.7 \%)(P$ 
$=0.037)$. Incidences of lung metastases was higher among patients with histological grade III/IV (56, $43.8 \%)(P<0.001)$. Patients with lung metastases had a larger tumor size $(49.96 \pm 90.25 \mathrm{~mm}, P<0.001)$. For each $10 \mathrm{~mm}$ increase in tumor size, the odds of having lung metastases increased by $6.6 \%$. Patients with lung metastases had a shorter survival time(HR:3.05, 95\% CI:2.17-4.28, $P<0.001)$. For age, tumor size increase and black study subjects were also significantly associated with a shorter survival time.

Conclusion: Our study provides insight into the epidemiology of lung metastases in patients with hypopharyngeal cancer. When the tumor is diagnosed, we should pay close attention to the sex, race, tumor size and histological grade in order to quickly detect the distant metastases.

Key Words: hypo-pharyngeal cancer; lung metastases; SEER

\section{Key Points:}

1. Regional metastases in hypo-pharyngeal cancer such as lymphatic metastases are easy to be found at the time of tumor diagnosed. Though there is a high incidence rate of lymphatic metastases; the detection of distant metastases is still rare in hypo-pharyngeal carcinoma.

2. Only $8.5 \%$ patients were observed to have distant metastases in head and neck squamous cell carcinoma. Among them, $16.3 \%$ of the patients suffered from hypo-pharyngeal carcinoma. Although distant metastases in hypo-pharyngeal carcinoma are not common, they play an important role.

3. Population-level estimates for prognosis among patients including newly diagnosed hypo-pharyngeal carcinoma and lung metastases or other organs' metastases are extremely rare.

4. Our study provides insight into the epidemiology of lung metastases in patients with newly diagnosed hypo-pharyngeal cancer.

5. When the tumor is diagnosed, we should pay close attention to the sex, race, tumor size and histological grade in order to quickly detect the distant metastases.

\section{Introduction}

Hypo-pharyngeal carcinoma is often difficult to be diagnosed in its early stages because of its occulted incidence site. Regional metastases such as lymphatic metastases are easy to be found at the time of tumor diagnosed ${ }^{1}$. Though there is a high incidence rate of lymphatic metastases; the detection of distant metastases is still rare in hypo-pharyngeal carcinoma.

Only $8.5 \%$ patients were observed to have distant metastases in head and neck squamous cell carcinoma. Among them, $16.3 \%$ of the patients suffered from hypo-pharyngeal carcinoma. The metastatic organs are relatively limited as reported: the lungs (77\%), bone (19\%), mediastinal and other lymph nodes (4\%), as well as the brain, liver ${ }^{1,2}$. Although distant metastases in hypo-pharyngeal carcinoma are not common, they play an important role. Lymphatic metastases are most often the cause of mortality due to hypo-pharyngeal carcinoma ${ }^{1}$.

Population-level estimates for prognosis among patients including newly diagnosed hypo-pharyngeal carcinoma and lung metastases or other organs' metastases are extremely rare, however the incidence of lung metastases or other organ metastases combined with hypo-pharyngeal carcinoma occurs less frequently than newly diagnosed hypo-pharyngeal carcinoma. Data was sourced from a single institution or case report. The purpose of this study was to use the Surveillance, Epidemiology, and End Results (SEER) database to characterize the incidence of lung metastases among patients with hypo-pharyngeal cancer on a populationbased level. We also quantified survival estimates and assessed other potential risk factors that influence survival among patients with hypo-pharyngeal cancer and lung metastases present at the time diagnosis.

\section{Methods}

\section{Study design and study population}

The SEER database is a premier source for cancer statistics in the US; it includes information on cancer incidence, treatment, and survival for approximately $30 \%$ of the US population. Information on the status of lung metastases in hypo-pharyngeal cancer from 2010 to 2014 at the time of initial cancer diagnosis was 
released in 2017. By using the SEER database, 2833 patients aged 20 years or older who were diagnosed with hypo-pharyngeal cancer between January 1, 2010, and December 31, 2014 were identified. Patients whose lung metastases status at time of diagnosis was either unknown or not applicable, or patients who didn't schedule a follow-up appointment were excluded, eventually leaving 2714 patients in the final cohort.

Reporting guideline: Secondary data analysis

\section{Statistical analysis}

A descriptive analysis was performed. Mean/standard deviation \& median/ interquartile range or frequency \& percentage were reported for continuous and categorical variables respectively. Comparisons of demographic and clinical characteristics by lung metastases status were conducted using Pearson Chi-square and Wilcoxon rank-sum tests appropriately.

The incidence of lung metastases among hypo-pharyngeal cancer patients was defined as the number of hypo-pharyngeal cancer patients diagnosed with lung metastases divided by the total number of patients with hypo-pharyngeal cancer. The 95\%CI of incidences was reported using the Wilson method. We also calculated incidences along with $95 \%$ CIs by age group, race, sex, histological grade, AJCC stage, tumor size by groups, metastases, and primary site.

A multivariable logistic regression model was conducted to evaluate the association between lung metastases and age, race, sex, histological grade, tumor size and primary site where both age and tumor size were treated as continuous covariates. Presence of bone, brain, or liver metastases at the time of cancer diagnosis is available in the SEER database and was used to characterize the extent of systemic disease among patients in this study.

In addition we performed a multivariable Cox Proportional Hazards regression model to estimate survival time between patients with or without lung metastases during a five year follow-up period while controlling for the covariates listed above. Subjects who died of other non- hypo-pharyngeal cancers and those who lost follow-up were censored.

Almost 35\% of patients were missing tumor sizes; as a result multiple imputation with 100 imputed datasets was performed. The results of these models with or without multiple imputation were consistent and complete case model results were reported.

All statistical analyses were performed using STATA statistical software, Version 16.0 (College Station, TX) and $\mathrm{R}$ version 3.5.0. Statistical significance was defined as two-sided alpha level at 5\%.

This study was approved by the institutional review board at Zhongshan Hospital of Fudan University; written and informed consent was waived.

\section{Results}

2833 patients who were diagnosed with a hypo-pharyngeal cancer between January 1, 2010, and December 31, 2014 were identified. Patients whose lung metastases status at diagnosis were unknown, not applicable or patients who didn't record follow-up times were excluded; leaving 2714 patients in the final cohort. The median age was 64 years (IQR: 57 to 73 years), $81.2 \%$ were men, the majority race was Caucasian (69.5\%) and the median follow-up time was 12 months (IQR: 5 to 26 months). Among them, $48.8 \%$ of patients' tumors occurred in the pyriform sinus, while $51.2 \%$ patients were in the post-cricoid region and posterior wall of the pharynx. The median tumor size was $30 \mathrm{~mm}$ (IQR: 21 to 44mm), and categorized into three groups: < 10 $\mathrm{mm}(1.7 \%), 10-30 \mathrm{~mm}(31.2 \%)$, and $>30 \mathrm{~mm}(32.2 \%)$. 171 patients were identified to have metastases at least in one site, such as lung $(128,4.7 \%)$, liver $(28,1.0 \%)$, bone $(46,1.7 \%)$ or brain $(7,0.3 \%)$. Among the entire cohort, most of the patients were grade II/III(1053,38.8\% / $884,32.6 \%)$ (Table 1).

\section{Incidence of lung metastases}

Of the 2714 test patients, 171 were identified to have distant metastases, of which 128 patients had lung metastases. Females were less likely to have lung metastases(15, 11.7\%) compared to male patients 
( $P=0.037)$. Patients with lung metastases had larger tumor sizes $(49.96 \pm 90.25 \mathrm{~mm})$ than the patients without(35.06 $\pm 23.16 \mathrm{~mm}, P<0.001)$. Tumors of grade III/IV were more likely to have lung metastases $(56$, $43.8 \%)$ than those with grade I/II $(P<0.001)$. Less follow-up time $(8.92 \pm 10.31, P<0.001)$ and higher mortality due to the first tumor $(74,57.8 \%)(P<0.001)$ was seen in patients with lung metastases. Age, race and primary tumor site was not significantly associated with lung metastases. Interestingly, patients with lung metastases were quite likely to have more than two organs with distant metastases $(31,24.2 \%)(P<0.001)$ (Table 1). The detailed incidence rates of lung metastases showed in Table 2.

Upon multivariable logistic regression (Table 3) among the entire cohort, females versus males' odds ratio $(0.4,95 \%$ CI: $0.17-0.93, P<0.001)$ was associated with significantly less odds of having lung metastases at diagnosis. Black and Hispanic people seem to incur a higher rate of lung metastases than other races (OR:1.91, 95\% CI: 1.03-3.53, $P=0.04$; OR:2.31, 95\% CI:1.16-4.62, $P=0.018$ ). Greater tumor sizes resulted in greater odds of lung metastases(OR :1.01, 95\% CI: 1.00-1.01, $P=0.01$ ) among entire cohort. For each10 mm increase in tumor size, the odds of having lung metastases increases by $6.6 \%$, controlling for other covariates. The predicted probability of lung metastases increased when tumor size increased (Figure 1).

\section{Survival}

Upon multivariable Cox regression for all causes of mortality among the entire cohort, patients with lung metastases had a shorter survival time than those without lung metastases(HR:3.05, 95\% CI:2.17-4.28, $P$ $<0.001)$. The variables: age (HR:1.01, 95\% CI: 1.29-3.67, $P=0.024)$, Tumor size increase (HR:1.00, $95 \%$ CI: 1.00-1.01, $P<0.001$ ), and race (African Americans) (HR:1.89, 95\% CI:1.49-2.40, $P<0.001$ ) were also significantly associated with a shorter survival time. Tumors located in the pyriform sinus(HR:0.83, $95 \%$ CI:0.69-1.00, $P=0.048$ ) indicates a longer survival time. (Table 4)

Patients in the survival cohort with hypo-pharyngeal cancer with or without lung metastases at diagnosis had a 5 year follow-up. After adjusting for sex, age, primary site of tumor and tumor size, the 5 year survival probability and cumulative mortality of patients with lung metastases was $20 \%$ and $80 \%$ respectively; the patients without lung metastases had 5 year survival probabilities and cumulative mortality rates of $60 \%$ and $40 \%$ (Figure 2). These results are consistent with the results of the cox regression. African Americans had a high cumulative mortality of $60 \%$, while white people only had a rate of $40 \%$ (Figure 3 ). The tumors' primary site of patient didn't display a significant difference in cumulative mortality (Figure 3).

\section{Discussion}

In this study, the incidence of lung metastases among patients with newly diagnosed hypo-pharyngeal cancer was calculated. We found that there was no significant difference in the incidence of lung metastases in the primary site of the tumor. Unsurprisingly, females were less likely to have lung metastases. Since many of the female patients' lung metastases status at diagnosis was unknown, not applicable or did not record follow-up times and these patients were excluded, the true incidence of lung metastases in female patients with hypopharyngeal cancer is likely underestimated by the results of this study. In addition, we are pleased to report for the first time that for each $10 \mathrm{~mm}$ increase in tumor size, the odds of having lung metastases increases by $6.6 \%$. This result may allow us to further predict the probability of lung metastases based on tumor size. High histological grade III/IV had the highest incidence of lung metastases in this study and was consistent with the common knowledge. Moreover, we discovered that patients with lung metastases were more likely to have other metastases. This suggests that when we find lung metastases, we should perform further tests such as: MRI, B-ultrasound and bone scan to exclude the possibility of other metastases. The development of PET-CT makes finding other metastases sites such as the aforementioned ones, much simpler.

Since hypo-pharyngeal cancer has a relatively lower incidence rate, research is scant on the topic of lung metastases of this cancer. Even research on head and neck squamous cell cancer is rare. Ampil, et al ${ }^{3}$. reported 37 patients with treated head and neck cancer had lung metastases, but did not mention the incidence rate. Marc Mareel, et al ${ }^{4}$ reported incidences of lung metastases in head and neck cancer patients at $10.8 \%$ of all the studied patients and $78 \%$ of all distant metastasis patients. They also found that the prevalence of distant metastases in hypo-pharyngeal cancer was $20.5 \%$. This result was somewhat different 
from our study. The incidence of lung metastases in the Mareel study was only $4.7 \%$ of all the studied patients and the incidence of all distant metastases was $6.3 \%$ in this study. This discrepancy may be because the Mareel study only sampled from a single center's study and did not focus on hypo-pharyngeal cancer. Our study using SEER data includes approximately $30 \%$ of the United States population, the incidence proportions we describe are highly generalizable and more likely to be reflective of the population compared to previously published data focused primarily on patients treated at academic cancer centers. Macherey, et $\mathrm{al}^{5}$. performed a meta-analysis/review of all papers published between 2000 and 2014; they found lung metastases develop in 1.9-13\% of head and neck cancer patients. The results of our study seem agree with Macherey's findings.

The study conducted by Marc Mareel, et al also showed $47 \%$ of patients died from their primary tumors during follow-up of which $52 \%$ of patients had distant metastases, however the tumor site and distant metastases site were not separated in Mareel's study. In our study only $28.1 \%$ of patients died as a result of their first tumor; of those individuals, $57.8 \%$ of patients had lung metastases. Compared to Mareel's study, our study focused on hypo-pharyngeal cancer and lung metastases; it can truly illustrate the effect lung metastases of hypo-pharyngeal cancer has on survival. P. Pracy, et al. reported the overall 5-year disease specific survival rate in hypo-pharyngeal cancer patients as approximately $30-35 \%$ in the United Kingdom. By reviewed articles of head and neck cancer, Macherey, et al. reports a $58 \% 5$-year survival rate ${ }^{5}$. In our study, $71.9 \%$ of patients were alive or succumbed to other causes instead of the first tumor after the 5-year follow-up. The reported 2-years over survival(OS) in head and neck cancer with distant metastases was $15 \%$ in Belgium, while in our study, survival was close to $40 \%$ in hypo-pharyngeal cancer patients with lung metastases and the survival probability gradually decreased to $20 \%$ after the 5 -year follow-up. Our results were slightly different from the studies in the United Kingdom and Belgium, because the population composition may be different in three countries. A study including a broader range of countries could account for this discrepancy ${ }^{4,6}$.

As is shown in previous studies, a lot of risk factors will affect survival time in head and neck cancer ${ }^{7}$. Among the entire cohort, although the tumor primary site in the pyriform sinus didn't have significantly lower odds of lung metastases, they indicate a lower risk associated with all-caused mortality (HR:0.83, 95\% CI:0.69-1.00, $P=0.048$ ). This suggests that the primary site of tumor is still worthy of further research. African Americans not only have significantly greater odds of lung metastases, but also suggests a higher risk associated with all-caused mortality(HR:1.89, 95\% CI:1.49-2.40, $P<0.001$ ). This finding presents a bad prognosis for African Americans who are diagnosed with hypo-pharyngeal cancer. Tumor size is an undoubted risk factor for mortality, for each $10 \mathrm{~mm}$ tumor size increase, the risk of death increases by $3.9 \%$. It suggests that a closer follow-up study is needed for patients with larger tumors.

Lung metastases is often caused by cancers of peritoneal organs, including gastric cancer, colon cancer, pancreatic/biliary cancer, ovarian cancer, and uterine cancer ${ }^{8}$. Most of these cases are adeno-carcinomas; only a few cases are derived from squamous cell cancer ${ }^{9-12}$. In contrast to these studies, we found more patients having lung metastases with newly diagnosed hypo-pharyngeal cancer. We revealed that race, histological grade and tumor size were associated with having lung metastases at diagnosis. In the future we should be wary of these factors.

\section{Limitations}

This study did not include data concerning the HPV status of patients. Positive HPV status may suggest a different prognosis for head and neck squamous cell carcinoma. Perhaps more importantly information regarding the treatment methods of patients was unavailable. Different treatment methods may affect the survival of patients. Finally, the SEER database includes only patients from the United States; different countries may result in different tumor prognosis. For the aforementioned reasons our findings may have resulted in an underestimation of our results.

\section{Conclusion}

Despite the above limitations, our study provides insight into the epidemiology of lung metastases in patients 
with newly diagnosed hypo-pharyngeal cancer in the United States. When the tumor is newly diagnosed, we should pay close attention to the sex, race, tumor size and histological grade in order to quickly detect distant metastases.

\section{Data Availability Statment}

The datasets generated during and/or analysed during the current study are available from the corresponding author on reasonable request.

\section{Conflicts of interest}

None.

\section{References}

1. Spector JG, Sessions DG, Haughey BH, et al. Delayed regional metastases, distant metastases, and second primary malignancies in squamous cell carcinomas of the larynx and hypopharynx.Laryngoscope. 2001;111(6):1079-1087.

2. Wakasaki T, Omori H, Sueyoshi S, et al. A case of peritoneal metastasis during treatment for hypopharyngeal squamous cell carcinoma. World J Surg Oncol. 2016;14(1):265.

3. Ampil F, Previgliano C, Porter C, Richards T, Takalkar A. Metachronous mediastinal and lung metastases from head and neck cancer: A case series, literature review and considerations for treatment. Oral Oncol. 2019:104518.

4. Duprez F, Berwouts D, De Neve W, et al. Distant metastases in head and neck cancer. Head Neck. 2017;39(9):1733-1743.

5. Macherey S PS, Doerr F, Grönke S, Heldwein M, Quaas A, Zander T, Hekmat K. Surgical therapy of lung metastases from head and neck cancer.HNO. 2015;62(12):9.

6. Pracy P, Loughran S, Good J, Parmar S, Goranova R. Hypopharyngeal cancer: United Kingdom National Multidisciplinary Guidelines. J Laryngol Otol. 2016;130(S2):S104-S110.

7. Pfister DG SS, Brizel DM, Burtness B, Busse PM, Caudell JJ, Cmelak AJ, Colevas AD, Dunphy F, Eisele DW, Foote RL, Gilbert J, Gillison ML, Haddad RI, Haughey BH, Hicks WL Jr, Hitchcock YJ, Jimeno A, Kies MS, Lydiatt WM, Maghami E, McCaffrey T, Mell LK, Mittal BB, Pinto HA, Ridge JA, Rodriguez CP, Samant S, Shah JP, Weber RS, Wolf GT, Worden F, Yom SS, McMillian N, Hughes M. Head and Neck Cancers, Version 1.2015.J Natl Compr Canc Netw. 2015;13(7):9.

8. Ayantunde AA, Parsons SL. Pattern and prognostic factors in patients with malignant ascites: a retrospective study. Ann Oncol.2007;18(5):945-949.

9. Madiba TE, Haffejee AA, Mbete DL. Squamous cell carcinoma of the oesophagus-an unusual presentation with malignant ascites. A case report. S Afr J Surg. 1993;31(4):137-139.

10. Beltran H, Robinson BD, Tagawa ST. Primary squamous cell carcinoma of the urinary bladder presenting as peritoneal carcinomatosis. Adv Urol. 2010:179250.

11. Yarmohammadi H, Mansoori B, Wong V, et al. Squamous cell carcinoma arising from ovarian mature cystic teratoma and causing small bowel obstruction. J Cancer Res Ther. 2014;10(3):770-772.

12. Wong ZW, Leong SS, Tan T, Mancer K. A case of metastatic squamous cell carcinoma of the hypopharynx manifesting as acute abdomen.Ann Acad Med Singapore. 2004;33(3):356-358.

Figure legends 
Figure 1. The predicted probability of lung metastases increased when tumor size . For each10 $\mathrm{mm}$ increase in tumor size, the odds of having lung metastases increases by $6.6 \%$, controlling for other covariates.

Figure 2. The cumulative mortality of patients with or without lung metastases. The patients with lung metastases had a higher rate of cumulative mortality.

Figure 3. The cumulative mortality of patients in different race and primary site. African Americans had a high cumulative mortality of $60 \%$, while white people only had a rate of $40 \%$ (Figure 3 ). The tumors' primary site of patient didn't display a significant difference in cumulative mortality.

\section{Hosted file}

Tables(1-4).pdf available at https://authorea.com/users/373437/articles/491130-lungmetastases-in-newly-diagnosed-hypo-pharyngeal-cancer-a-population-based-study

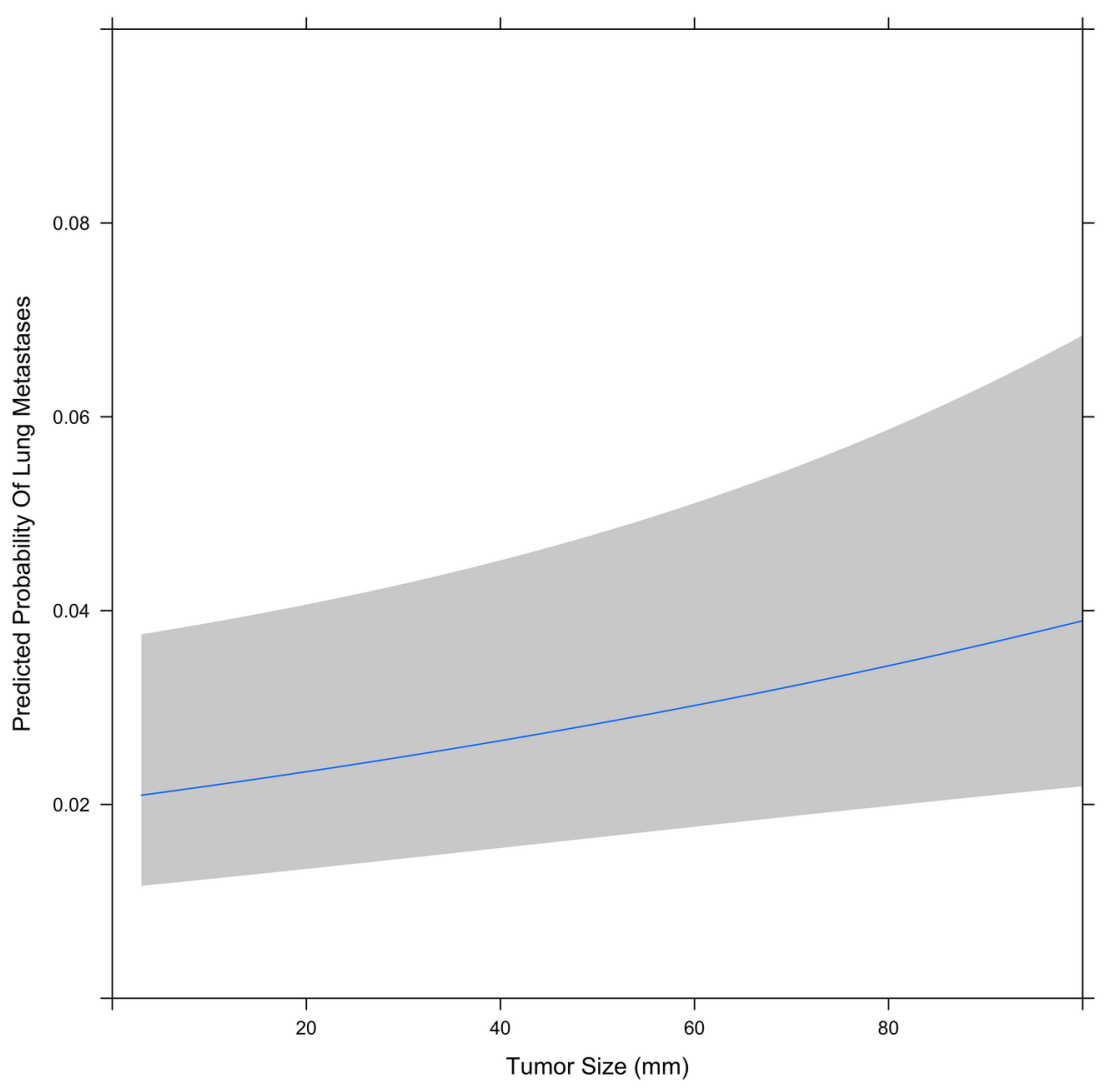




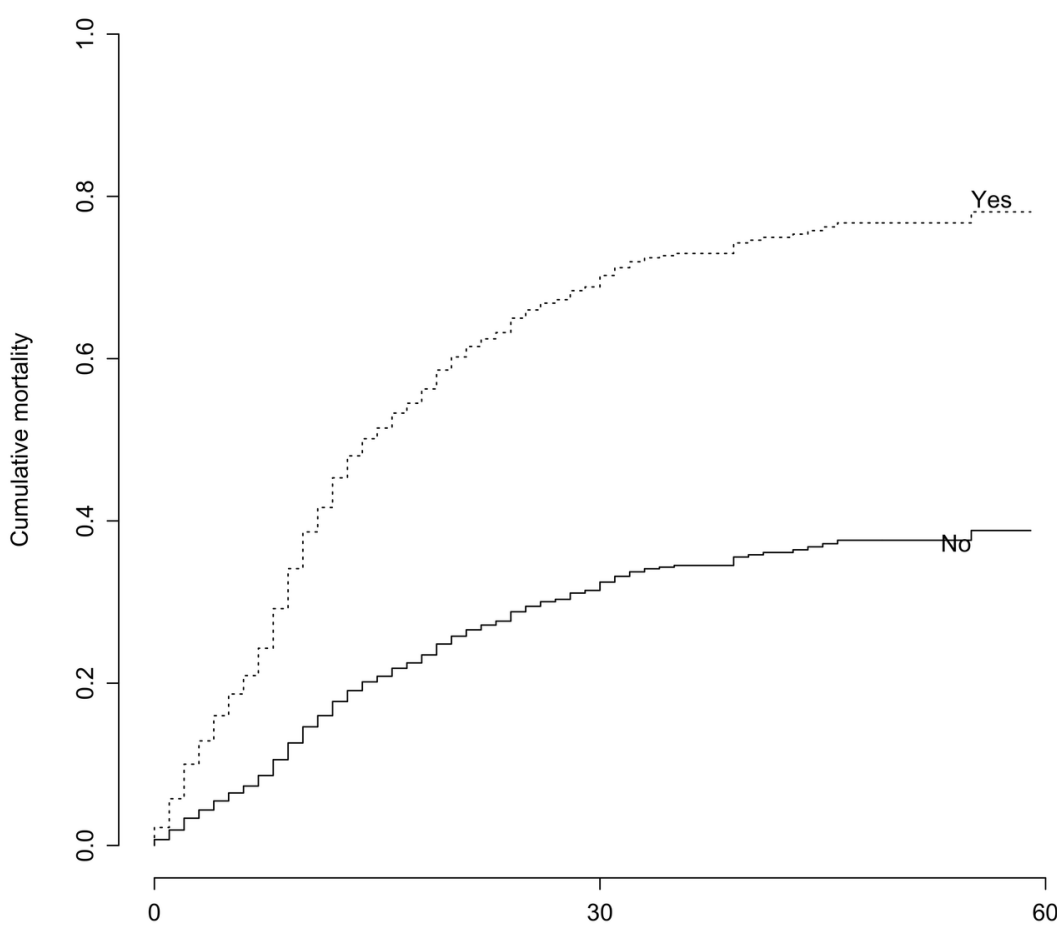

Follow-up time in months 

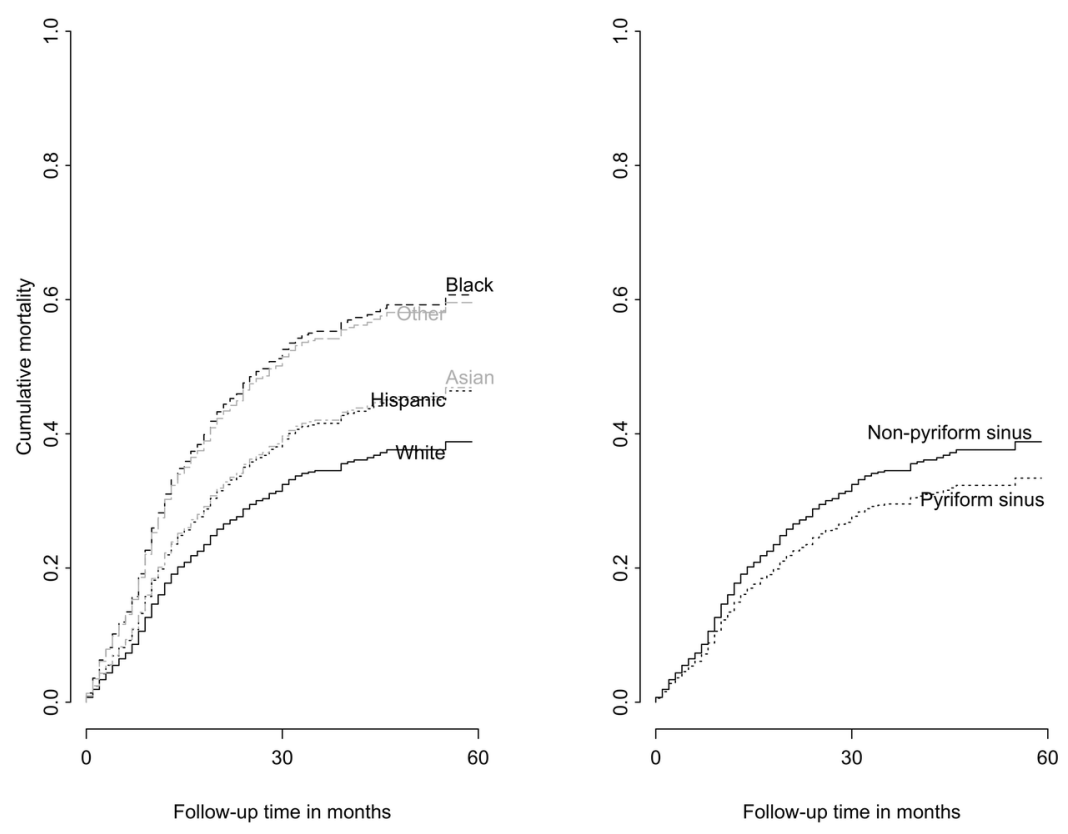